DOI: $10.21608 / z v i z .2017 .29241$.

\title{
Evaluation of Carbon Fiber and Bovine Pericardium Implantation in Tenorrhaphy of Common Calcanean Tendon Rupture in Goats
}

Ali E. Kandeel ${ }^{1,2}$, Mahmoud A. Abdraboh ${ }^{1}$, Abd El- Basit M. Abd El Aal ${ }^{1}$ and Shimaa A. Ezzeldein $^{1 *}$

${ }^{1}$ Surgery, Radiology and Anesthesiology Department, Faculty of Veterinary Medicine, Zagazig University, 44511, Egypt

${ }^{2}$ Biology Department, Faculty of Sciences and Arts, University of Jeddah, Alkamil Branch, KSA

\section{Abstract}

Common calcanean tenorrhaphy was carried out on right hind limbs of 18 goats after removal of two $\mathrm{cm}$ segment from the tendon using linen (nylon) No. one and locking loop suture pattern alone (group I) or together with carbon fibers implantation (group II) or with bovine pericardial flap (group III). Surgery was done under epidural analgesia and the tibiotarsal joint of the operated limb was immobilized using cortical screw $3.5 \mathrm{~mm}$ with application of full limb plaster cast. Post-operative healing of the tendon was monitored through clinical observation, ultrasonographic, macroscopic and microscopic examinations at intervals (3, 9, 12 weeks) post operatively. With the use of bovine pericardial flap, the operated animals showed earlier improvement compared with the others. Bovine pericardial flap also induced early tendon healing and better organization of tendon fibers.

Keywords: Common Calcanean Tendon Rupture, Tenorrhaphy, Goats, Bovine Pericardium.

\section{Introduction}

Tendons are an important constituent of the musculoskeletal system through which energy is transmitted, distributed and expressed in the form of movements. Common calcanean tendon is one of the largest tendons of the animal body and is known as Achilles tendon. In small ruminants, the term Achilles tendon is commonly used among clinicians for all structures inserted in the Os-calcis. This tendon consisted of medial and lateral heads of muscle gastrocnemius and soleus, muscle superficial digital flexor, muscle gluteobiceps, muscle gracillis as well as muscle semitendinosus [1].

The primary function of this tendon is to extend the tibiotarsal joint and flex the stifle and digits [2,3]. Common calcanean tendon affections ranged from spontaneous rupture to acute or chronic tendonitis. In small animals, the disruption or displacement of the Achilles tendon with fracture of the calcaneus bone, displacement of the superficial digital flexor tendon, musculo-tendinious tear and avulsion head of gastrocnemius muscle are the recorded affections [4,5]. By rupture of this tendon tarsal hyper flexion and stifle hyper extension occurs on bearing weight on the affected limb, in addition to change of posture and flaccidity of hock. The degree of lameness is dependent on the severed components of the common calcanean tendon in small ruminants [3, 4]. Ultrasonography has been a popular technique for the diagnosis of Achilles tendon rupture and has been used also in the follow up of treatment $[6,7,8]$. The suture material of choice for tenorrhaphy should be strong and inert [9]. Suture pattern selected should minimize ischemia of the tendon and resist gap $[10,11]$. Carbon fibers act as a scaffold which encourage the formation of new tendinous tissue in nearly normal orientation and good tensile strength, where the connective tissue and collagen fibers invade the implant and grow among the carbon filaments [12, 13]. The bovine pericardium used in reconstruction of digital extensor tendons in an ovine model as the tendon-graft junctions were augmented and shielded from surrounding tissues by chemically-treated bovine pericardium [14]. Tendon elongation and augmentation were done also with bovine pericardium $[15,16]$. This work aimed to, clinical and ultrasonographic comparative evaluation of tenorrhaphy with carbon fiber or bovine pericardial flap implantation in complete rupture of common calcanean tendon (Achilles tendon) in goats with immobilization of the tibiotarsal joint.

\footnotetext{
*Corresponding author email: (shimaa.ezzeldein@yahoo.com), Surgery, Radiology and Anesthesiology 


\section{Material and Methods}

The present study was carried out on 18 clinically healthy goats of different ages (10 to 18 months old), with body weights ranged between $15-25 \mathrm{~kg}$. The animals were housed and managed in the isolation stable, Faculty of Veterinary Medicine, Zagazig University. All the operated animals were prepared for surgery. Food was withheld 12 hours preoperatively while water prevented 6 hours preoperatively. The animals were given broad spectrum antibiotic (Epicocillin, Egyptian International Pharmaceutical Industries. Co. $10^{\text {th }}$ Ramadan City Egypt.) in a dose of $0.5 \mathrm{gm} / 24$ hours before surgery through intramuscular injection for five successive days, and were also subcutaneously injected with Anti titanic serum, VACSERA, A.R.E $1500 \mathrm{I} / \mathrm{U}$. The surgical operations were done under epidural analgesia (lumbosacral injection), using $1 \mathrm{ml} / 4 \mathrm{~kg}$ lidocaine $2 \%$ (Debocaine. Debaky Pharm., Egypt) in standing position. Each animal was fixed on the operation table in the lateral recumbence with the upper hind limb unfixed to be operated. The operative site was prepared aseptically. A lateral skin incision was made seven centimeters proximal to the os calcis. Blunt dissection of the skin from the underlying subcutaneous tissue exposing the common calcanean tendon and os calcis was done. Two centimeters segment from the tendinious part (middle third) of the tendon was severed. The animals were classified into three groups each of six animals using three different methods for Achilles tendon tenorrhaphy. In (Group I) tenorrhaphy was done with locking loop suture pattern using nylon no1 (Linen, Kruuse sutures Kruuse) (Fig.1a). In (Group II) tenorrhaphy was done using linen (nylon) no.1in addition to carbon fibers (Grafil, courtaulds limited,carbon fiber division Po. Box 16 Coventry,England) implantation in the gap between the two cut ends(Fig.1b). Carbon filaments were braided washed for two minutes with acetone to remove any sizing polymer and steam sterilized before implantation. In (Group III) tenorrhaphy was done using linen (nylon) |No. 1 , in addition to bovine pericardial flap implantation (Fig.1c). The pericardia were cut away from the heart, put in sterile normal saline and shaken for 60 minutes, then transferred to a sterile glass bottle containing $50 \%$ sterile glycerol under aseptic condition and kept there for three hours at room temperature, then disinfected in $0.05 \%$ sodium hypochlorite and shaken in sterile normal saline after [17].

Tibiotarsal joint immobilization was done in all goats with compression screw stabilization technique. Cortical screw $3.5 \mathrm{~mm}$ in diameter, 4-6 cm length was applied between the tuber calcis and the tibia with the use of bone drill and drill bit $2.7 \mathrm{~mm}$ in diameter (Fig.2). The screw was removed one week before tendon histopathological sampling for clinical evaluation of the operated limb and the gate of the animal was observed. The Achilles tendon was palpated for its continuity.

Suturing of the linear incision of the superficial digital flexor tendon with simple interrupted suture pattern using chromic cat gut no.0 was done. The wound was infiltrated with Ampicillin solution (Chemical industries development "CID"). The skin was closed with silk no. 1 using simple interrupted suture. An X-ray film was taken to ensure the correct application of the cortical screw (Fig.3).Application of full limb plaster cast was performed in all operated goats. The full limb plaster cast was removed after 10 days to remove the stitches and full limb bandage applied till the 21th day.

Post-operative healing of the Achilles tendon was monitored using clinical observation at the 10th day after stitches removal and at the different intervals 3, 9, 12 weeks postoperatively, Ultrasonography with linear transducer $8 \mathrm{MHz}$, gross and histopathological examination (two goats of each group were done at intervals 3, 9, 12 weeks post operatively).

A tendon biopsy was taken in buffered formalin solution $10 \%$ and proceeds the routinely paraffin section technique, and stained with Hematoxylin and Eosin stain [18]. Sections were assessed for inflammatory and fibroblastic activity in addition to orientation and maturation of the collagen. 


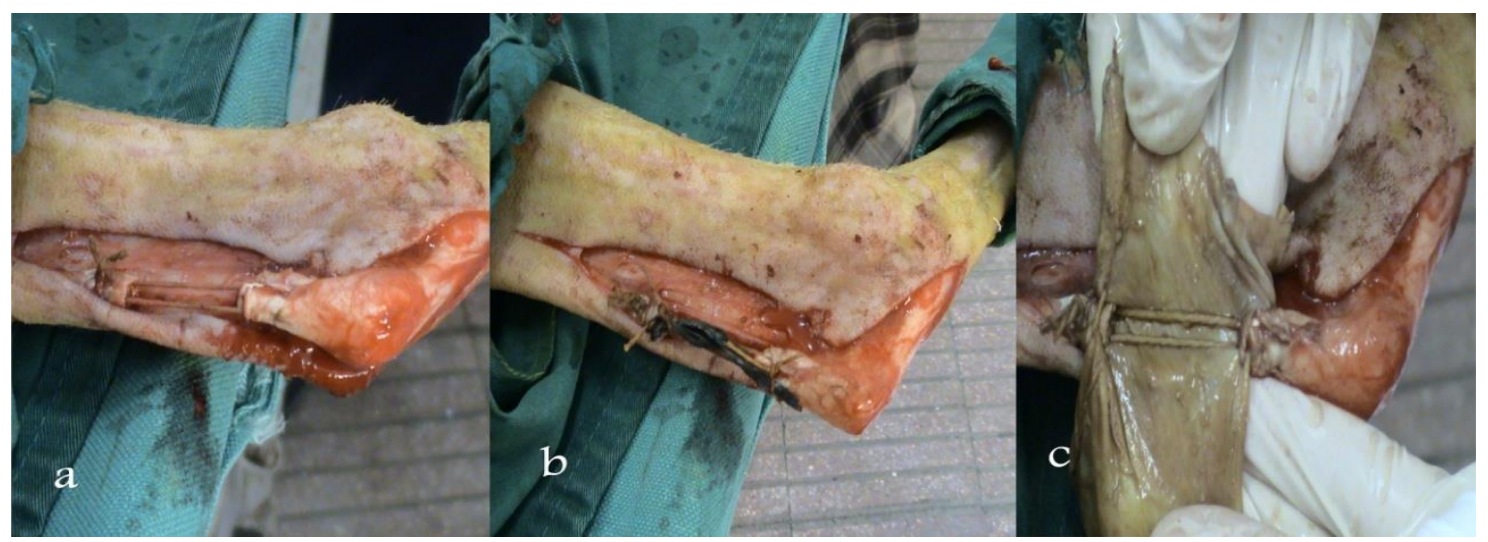

Figure 1: Showing suturing the common calcanean tendon of right hind limb with locking loop suture pattern using nylon only a, (Group I), nylon with carbon fiber b. (Group II), and nylon with pericardial flap c (Group III).

\section{Results}

Ten days post operation, the tendon gap was palpable in group I and II but in group III the gap couldn't be palpated. The inflammatory signs were more intense in group III in comparison to group I and II. The gliding movement of tendon was absent in all groups. Three weeks post operatively, subsiding of the cardinal signs of the inflammation was evident in all operated groups. Tenderness and adhesion of skin with the tendon defect were clearly seen in group I and II than group III. Non-weight bearing lameness was evident in group III. Landing the ground by the toe with severe lameness was evident in groups I and II. Soft thickening of the tendon defect was palpable in all groups. Gliding movement of tendon was evident in group III. Nine weeks post operation, tenderness and thickening were evident in group I and II. Thickening without tenderness was seen in group III. Landing the ground by the toe with severe lameness was evident in group III. Light degree of lameness was recorded in group III. The gliding movement of the tendon under the skin was palpable in group II and III but in group II tight adhesion of the tendon defect with the skin was still present. Twelve weeks post operation, animals walked normally without hyper extension or hyper flexion of the hock joint of the operated limb in group II and III. Light degree of lameness was recorded in all animals of group I. The repaired defect was thicker than remaining normal tendon in group II followed by group I. The gliding movement of tendon was normal without adhesion with the skin in group II and III. Skin adhesion with the repaired defect was still present in all animals of group I.
Figure 2: Showing application of screw with tenorrhaphy of right hind limb with locking loop suture pattern using nylon only (Group I).

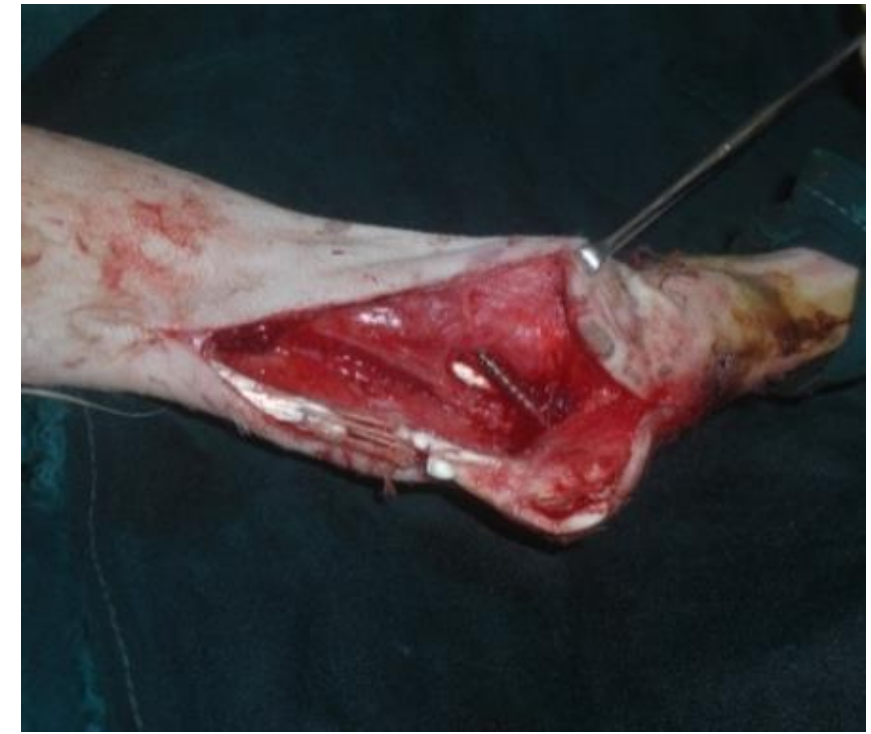


With ultrasonography, three weeks post operatively; In Group I, Fibrillar pattern of the tendon was lost. There were hypoechoic areas in between the area of hyperechogenicity. Excessive adhesions with the skin and peritendinous tissue occurred (represented by hyperechogenicity at the repaired site). The tendon thickness was increased with illdefined contour (Fig4a). By the $9^{\text {th }}$ week, the hypoechogenic inflammatory areas were decreased, the line of demarcation of the internal structure of the tendon still not clearly defined. Severe adhesions represented by hyperechogenicity of the tendon with the peritendinious tissue. The abnormal tendon thickness increasing began to decrease but still thickened than normal (Fig.4d). The sonogram showed nearly normal mottled hypoechoic to hyperechoic tendon texture, with moderate adhesions and less thickening than normal, at the $12^{\text {th }}$ postoperative week (Fig. 4 g).

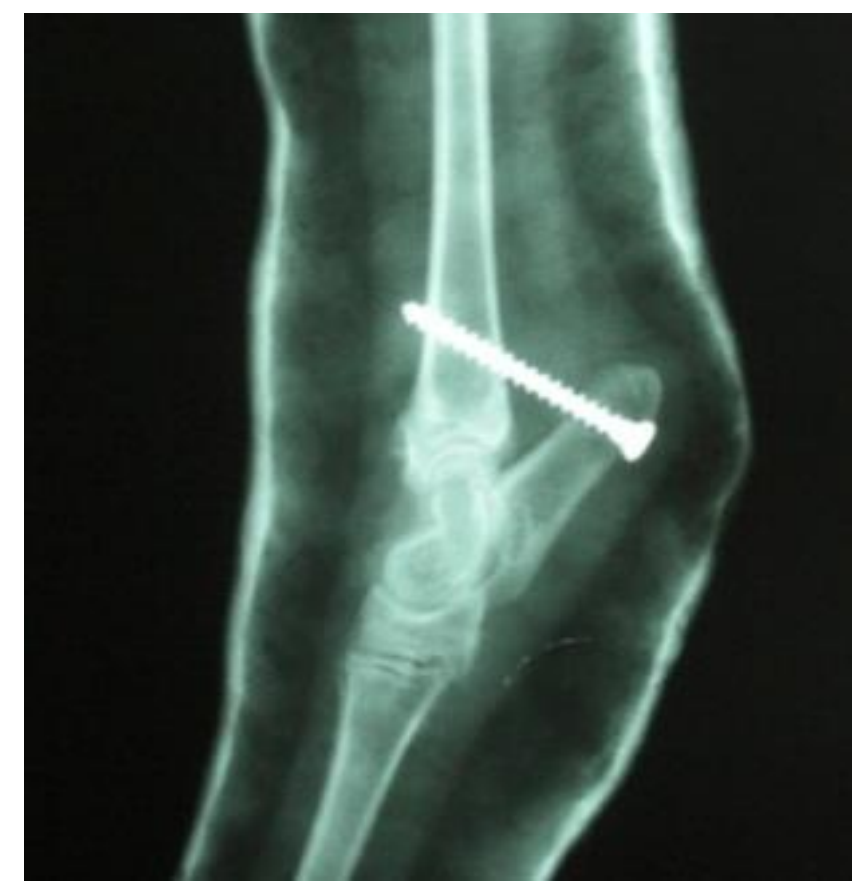

Figure 3: Showing $x$ ray film to confirm the presence of the cortical screw in tibiotarsal articulation for immobilization. 


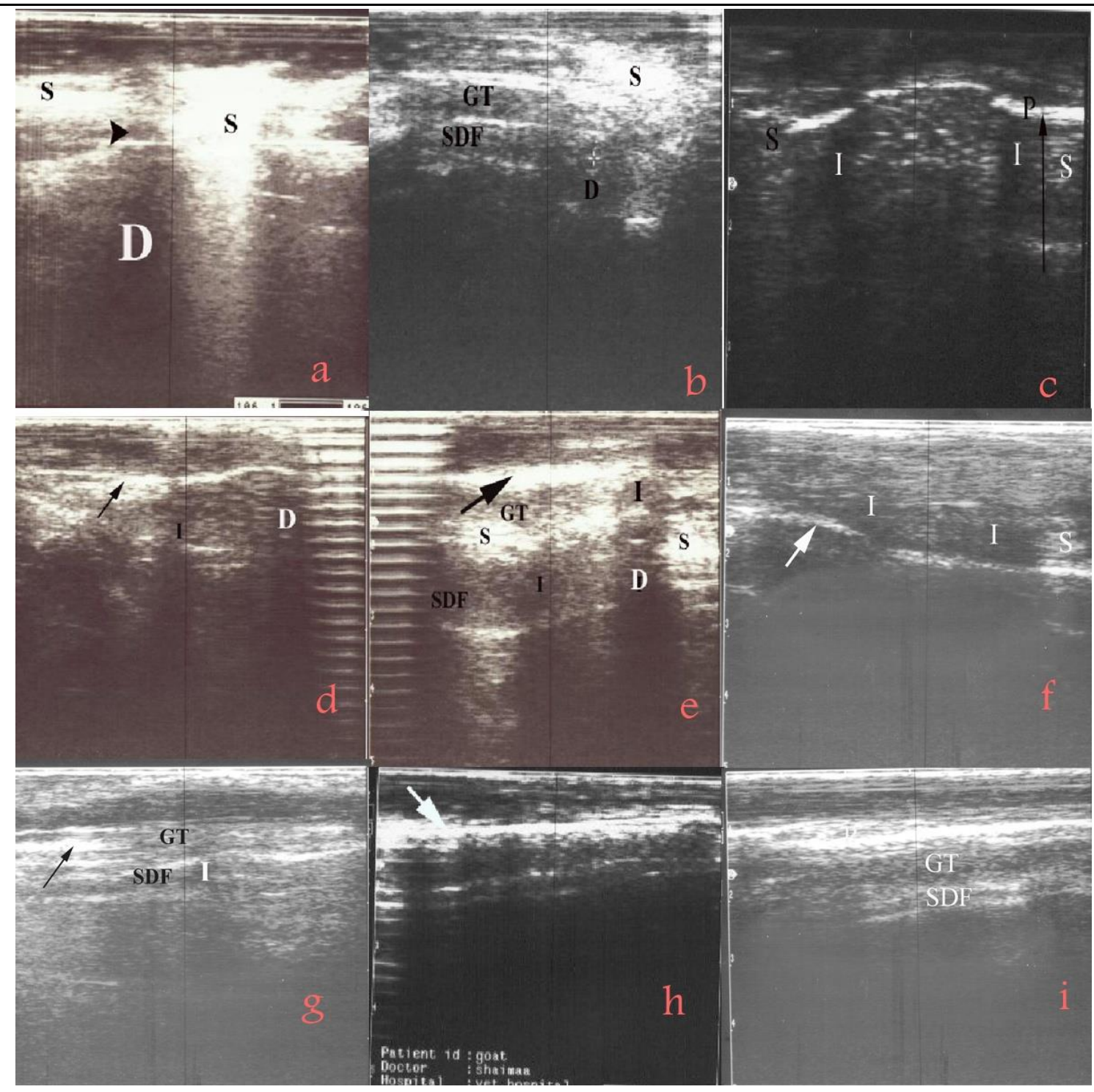

Figure 4: Ultrasonographic picture of common calcanean tendon of all the operated goats (Group I a, Group II b, Group III c at 3 weeks postoperatively), (Group I d, Group II e, Group III f at 9 weeks postoperatively) (Group I g, Group II h, Group III i at 12 weeks postoperatively). The figures showing: Excessive hyper echogenecity especially at the site of anastomosis (S). Distal shadowing (D) due to excessive hyperechogenicity. Inflammatory reaction as hypoechic areas (I). Arrow head: hypoechoic areas White or black Arrow: Adhesions. GT: Gastrocnemius tendon. SDF: superficial digital flexor tendon

In Group II, Tendon orientation was more clearly normal except at the site of anastomosis with carbon fibers and nylon that had more hyperechogenicity and loss of orientation with distal shadowing. The line of demarcation between the gastrocnemius tendon and the superficial digital flexor tendon was more defined. Hypoechogenic areas were noticed. Severe adhesions represented by hyperechogenic areas with distal shadowing were noticed more than that in group I at the same stage. The tendon at the area of implanted carbon fibers was increased than that of the contralateral limb with few millimeters (Fig.4b). With the $9^{\text {th }}$ week, tendon echogenicity was more hyperechogenic, and the tendon orientation was better and near to the normal. There were hypoecogenic areas 
within the tendinious tissue. Slight adhesions between the tendon and the other soft tissues were noticed. The internal structure of the tendon could be differentiated. The thickness of the tendon was increased (Fig.4e). The same observations of group I at the $12^{\text {th }}$ postoperative week was recorded (Fig.4h). In Group III, the sonogram revealed that, the echogenicity of the repaired site of the Achilles tendon was better than that in group II and III and the tendon was of normal hyperechogenicity. The tendon fibrillar pattern was like that in the normal non-operated limb in the same animal except at the site of anastomosis with the pericardial flap was more hyperechogenic with anechoic areas which may be due to inflammatory reaction. There were minimal adhesions between the tendon and the other soft tissues in the surroundings. There was slight increase in thickness, which was less than that in group II and more than that in group III (Fig.4c). With the $9^{\text {th }}$ week, the tendon echogenicity was going to the normal. The tendon orientation was normal with local areas of hypoechogenicity. There were hyperechogenic areas at the site of anastomosis. Slight adhesions were noticed and the tendon thickness was normal. There was slight increase in thickness (Fig.4f). The tendon appeared normal at the $12^{\text {th }}$ postoperative week (Fig.4i).

Histopathologically, 3 weeks post operatively. In group I, there was leukocytic infiltration. Hemorrhage and edema were adjacent the nylon fibers. Perivascular leukocytic infiltration mainly lymphocytes and macrophages was also noticed. Foreign body giant cells were observed inside the operated area. The nylon fibers were partially absorbed with macrophages and become faint in color (Fig.5a). In group II, The carbon fiber filled the cutting tendon. Leukocytic infiltrations were noticed at the site of the implanted carbon fiber. These leukocytic infiltrations were mainly neutrophils and few macrophages. There were extravasated erythrocytes seen among the tenocytes adjacent to the operated area. Edema around the implanted carbon fiber, serofibrinous exudates with inflammatory cells (macrophages) was noticed. Foreign body giant cells were observed inside the operated area (Fig.5b). In Group III, there were congested blood vessels, hemorrhage and leukocyte infiltration adjacent to the area of implanted pericardium. At the area of implanted pericardium there were serofibrinous exudates with lymphocytes and few neutrophils replaced the area of implanted pericardium. Edema infiltered with inflammatory cells mainly macrophages at the area of implanted pericardium flap which represented by empty space or homogenous pale eosinophilic substance were infiltrated with inflammatory cells mainly macrophages (Fig. 5c). 


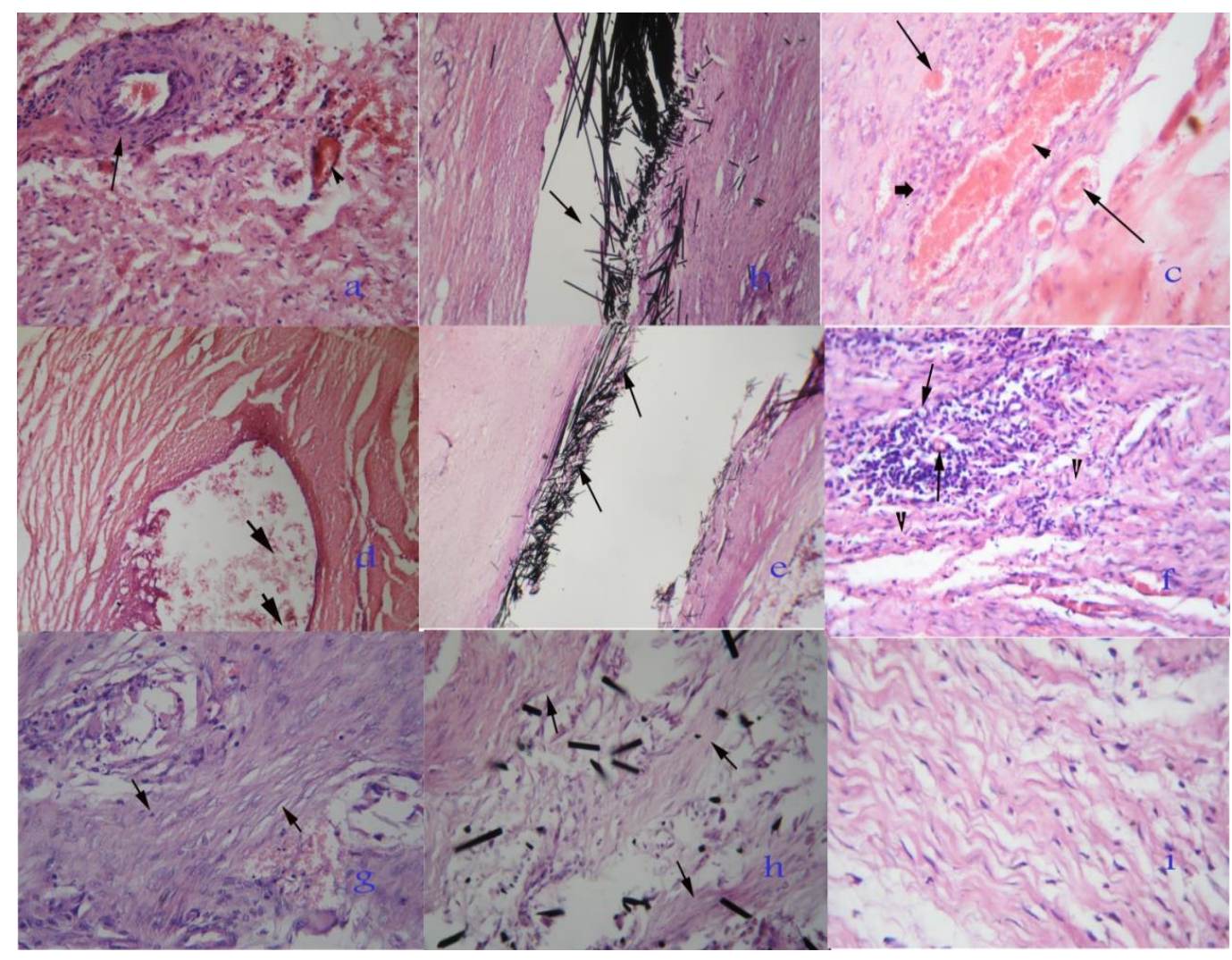

Figure 5: Histopathological image of a right Achilles tendon of all operated animals postoperatively showing: at 3 weeks postoperatively: Perivascular leukocyte infiltration(Arrow) mainly lymphocyte and macrophages beside congested hemorrhage among tenocyte (arrow head) with $H \& E \times 1200$ in Group I a. Edema in the area of implanted carbon fiber as serofibrinous exudate with inflammatory cell adjacent to the operated area with $(H \& E x 150)$ in Group II b. Serofibrinous exudates at the area of implanted pericardium infiltrated with inflammatory cells mainly macrophages with $(H \& E x 150)$ in Group III c. At 9 weeks postoperatively, Deposition of fibrin at area of operation with H\&Ex150 in Group I d. Increase the fibrin deposition and partially replace the carbon fibers with. H\&E X150 and Angioblasts were seen around the carbon fiber and the fibroblast in Group II e. The Pericardium flap is phagocytosed by macrophages, Angioblasts (arrow), and tenoblasts (arrow head) with lymphocytes. H\&EX 1200 in Group III f. At 12 weeks postoperatively: The area of nylon fiber is nearly completely filled with tenocyte with collagen fiber with H\&Ex1200 in Group I g. Regenerated tendon fibers which deposited haphazardly and irregularly with other areas of regular regenerated tendon which are parallel to the normal tendon was seen with H\&E x1200 in Group II h. Regenerated tenocytes in the same directions with H\&E X1200 in Group III i.

With the $9^{\text {th }}$ post-operative week, the nylon fibers were still present. Fibrin deposition was seen at the area of operation (Fig.5d). The fibrin depositions were increased with nearly absence of leukocytic activity partially replaced the carbon fibers. The carbon fibers were partially phagocytosed by macrophage. The angioblasts were found around the carbon fibers and the fibroblasts (Fig.5e). The pericardium flap was phagocytosed by macrophages, was seen as remnant of pericardium flap among the macrophages.
Angioblasts and tenoblasts were seen with the lymphocytes (Fig.5f).

With the $12^{\text {th }}$ week, the area of implanted nylon fiber was nearly completely filled with tenocytes with collagen fibers (Fig.5g). There were regenerated tendon fibers which deposited haphazardly and irregularly as tenoblasts around the carbon fibers. The regeneration of tendon fibers among new tendon replaced the operation empty area parallel to the normal tendon fibers. Regular regenerated tendon which was parallel to the 
normal tendon (Fig.5h). With the use of bovine pericardium, regenerated tenocytes in different directions were seen and in some areas the regenerated tenocytes were seen in the same direction (Fig.5i).

\section{Discussion}

In the present work, common calcanean tenorrhaphy was performed successfully on right hind limbs of goats after the removal of two $\mathrm{cm}$ segment from the tendon. The obtained results were similar to that mentioned by Worth et al. [19] who applied surgical repair of common calcanean tendon injuries in dogs.

The locking loop suture pattern using nylon material gave good stability without dehiscence in all operated groups. Nylon suture material provided good results in the repair of tendon defect; this finding may be attributed to that, nylon cause the least cellular response, strong and non-reactive. These results were in agreement with that previously recorded [9, 11]. The locking loop suture pattern was found to be satisfactory for tendon repair. Similar finding was stated by Worth et al. [19] in cats, [20] in dogs and [21] in goats. They revealed that the effectiveness of the tendon sutures is influenced by the transverse stability of the tendon and direction which help to protect against dehiscence of anastmosis and disruption of revascularization.

Immobilization of the hock in semi extention position by the application of compression screw stabilization technique using cortical screw for fixation of os calcis with the distal tibia had been given good immobilization of the common calcanean tendon in all groups, alike findings were reported by former authors [4, 19, 22, 23] who stated that, immobilization of the hock joint by position of screw between the tuber calcanei and distal part of the tibia proved a good results in the repair of common calcanean tendon in goats as early good immobilization was very essential to tendon repair and decrease adhesion.

The plaster cast was used to protect the operative area and minimizing edema in all groups. This was analogous to that performed by El Sharaby et al. [3].
The ultrasonography at 3 weeks showed sever inflammation in all operated goats of group I, this was manifested by hypoechoic areas in between the area of hyperechogenicity, as declared earlier by Maiti et al. [21].

At 9 weeks, clinically the goats landing the ground by the toe with sever lameness in all animals of group I, this confirms that the healing was improved by time. The sonogram showed increase in echogenecity of the tendon defect with evidence of hypoechogenic areas and fibrous proliferation as likely evidenced by Maiti et al. [21].

At 12 weeks, slight degree of lameness was present. The sonogram showed hypoechogenic area among the hyperechogenic tendon texture, and the longitudinal orientation of the tendon fiber was normal. Skin adhesion with the repaired defect was still present in all animals of group II; these results were due to incomplete healing of Achilles tendon repaired with nylon. Comparable results were indicated by former researchers [21, 22]. Bovine pericardium was preferable and indicated for grafting in tendons and ligaments, these results settled with other findings [14, 24-26].

\section{Conclusion}

The outcome of the present study confirms the superiority of glycerolized bovine pericardium graft over carbon fiber implantation and nylon tenorrhaphy alone.

\section{Conflict of interest}

The authors have no any conflict of interest.

\section{References}

[1] Getty, R. (1975): Ruminant myology. In Getty,R.; Sisson and Grossman`s. The anatomy of domestic animals 5 th ed. Chapter28 Pp. 849 -860. W.B. Saunders Co. Philadelphia, London, Toronto.

[2] Evans, E. H. (1993): Miller`s Anatomy of the dog, 3rd ed. Chapter6. Pp 357,369,377. W.B. Saunders, Philadelphia, London, Toronto, Montreal, Sydney, Tokyo.

[3] El Sharaby, A. A.; El Sayad, G. A. and Hammoda, A. K. (1998): Experimental rupture of the common calcaneal tendon in small ruminants. A surgical anatomical 
study with special reference to regional analgesia of the hind limb. 4th Scientific Vet. Med. Conference Zag.Univ.528-535

[4] Bloomberg, M. (1993): Muscles and Tendons in Slater, D. Text Book of small animal surgery. Chapter 146. Pp.20002013.

[5] Fenwick, S. P.; Hazleman, B. L. and Riley, G. P. (2002): The vasculature and its role in the damaged and healing tendon. Arthritis research. Vol. 4: No.4: 252-260.

[6] Leppilahti and Orava, S. (1998): Total Achilles tendon rupture. A review. Sports Med. 25(2)79-100.

[7] Kramer, M.; Gerwing, M.; Michele,V.; Schimke, E. and Kindler,S. (2001): Ultrasonographic examination of injuries to Achilles tendon in dogs and cats. J. Small anim. Prac., 42 (11) :531-535.

[8] Lamb, C. R. and Allice Duvernois (2005): Ultrasonographic anatomy of the normal canine calcaneal tendon. J. Veterinary radiology \&Ultrasound, Vol 46 (4): 326330.

[9] Keyhani, S.; Mardani-Kivi, M.; Abbasian, M.; Tehrani M. E. M. and Lahiji F.A. , 2013: Achilles tendon Repair, A Modified Technique. Research article. Arch Bone Joint Surg. 2013; 1(2): 86-89.

[10] Rawson, S.; Cartmell, S. and Wong, J. (2013): Suture techniques for tendon repair; a comparative review Muscles, Ligaments and Tendons Journal; 3 (3): 220-228.

[11] Behery, A. E.; Abd-El Aal, A.M. and Kandeel, A. E. (2008): Prognostic evaluation and treatment of some tendons and ligament lacerations in equine 9th Vet.Med.Zag. Conference. Port-said. 374-387.

[12] El Seddawy ,F. D. and ElShair,M.A.(1987): Surgical approach for the correction of carbon fiber implantation (case report). Zagazig Vet. J.XV.No. 2. 237-252.

[13] El Seddawy, F. D.; Kandeel, A. E. and Abd-El Maboud, M. (1994): Surgical management for traumatically divided flexor tendons in equines, and cattle with special reference to carbon fiber implantation.2nd Vet. Med. Cong. Zagazig. 217-230

[14] Rogers, G. J.; Milthorpe, B. K. Schindhelm, K.; Howlett, C. R. and Roe, S. (1995): Shielding of augmented tendon-tendon repair. Biomaterials J.Vol 16: page 803-807.

[15] Rossouw, P. and de villiers M. (2005): Bovine pericardial ligament and tendon augmentation: a new and revolutionary ligament. Journal of bone \& joint surgery, British volume, 87(supp iii), 277-277.

[16] Van Rijn, L. J.; Van De Ven, S. J.; Krijnen, J. S.; Jansen, S.M.; Bakels, A. J. and Lange horst A. M.( 2016): Tendon elongation with bovine pericardium (Tutopatch) when conventional strabismus surgery is not possible. Eur $\mathbf{J}$ Ophthalmol. 2016 May-Jun;26 (3):193202.

[17] Zuki, A. B. Z.; Hafeez, Y.M.; Loqman M.Y.; Noordin M.M. and Norimah,Y. (2007): Effect of preservation methods on the performance of bovine pericardium graft in a rat model. Anat.Histol. Embyol. 36,349-336.

[18] Suvorna, S. K.; Layton C. and Bancraft, J. D. (2013): Bancraft's Theory and Practice of histological techniques. 7thed. Curchill, Living Stone, Elsevier, England.

[19] Worth, A. J.; Danilsson, F.; Bray, J. P.; Burbidge H.M. and Bruce, W. J. (2004): Ability to work and owner satisfaction following surgical repair of common calcanean tendon injuries in work in dogs in New Zealand N.Z. Vet.J.52(3):109116.

[20] Milton, J. L. And Henderson, R. A. (1983): Muscles and Tendons. In Bojrab, J. M. Current techniques in small animal surgery. 2rd ed. Chapter 38. Pp. 507-509. Lea \& Febiger. Philadelphia, PA. USA.

[21] Maiti, S. K.; Kummar, N.; Singh, G. R.; Hoque, M. and Singh, R., (2006): Ultrasound therapy in tendinous injury healing in goats. J. Vet. Med. A 53, 249258 . 
[22] Saini, N. S.; Roy, K. S.; Bansal, P. S.; Singh, B. and Simran, P. S. (2002): A preliminary study on the effect of ultrasound therapy on the healing of surgically severed Achilles tendon in five dogs. J. Vet. med. A. 49:321-328.

[23] Abd El-Aal, A. M (1999): Autologus tensor fasciae lata as a donor for tendon grafting in dogs. J. Egypt vet. Med. Assoc. 59 No. 2\&3: 679-691.

[24] Chvapil, M.; Gibeault, D. and Wang, T. (1995): Use of chemically purified and cross-linked bovine pericardium .21: (12) 1383-1393.
[25] Gigante, A.; Cesari, E.; Busilacchi, A.; Manzotti, S.; Kyriakidou, K., Greco, F., Primio, R. D. and Mattioliae Belmonte M., (2009): Collagen I membranes for tendon repair: effect of collagen fiber orientation on cell behavior. Journal of Orthopaedic Research, 27(6), 826-832.

[26] Athar, Y.; Zainuddin, S. L. A.,; Berahim, Z.,; Hassan, A.; Sagheer, A. and Alam, M. K., (2014): Bovine Pericardium: A Highly Versatile Graft Material International Medical Journal Vol. 21, No. 3, pp. 321 - 324, Jun

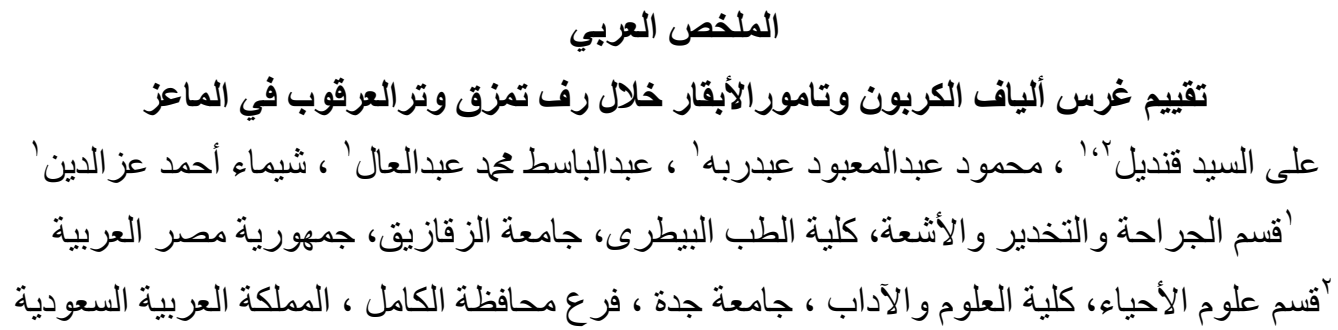

أجريت جراحة رف وتر العرقوب المشترك للقوائم اليمنى الخلفية لعدد 1/ معزة ، بعد إزالة جزء بطول سنتيمترين،

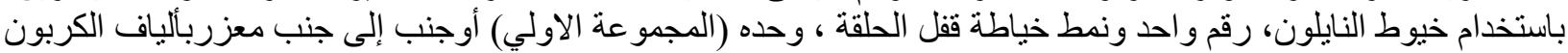

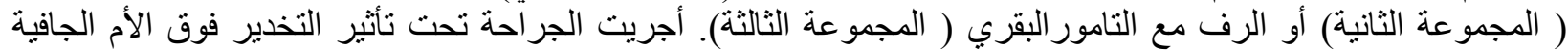

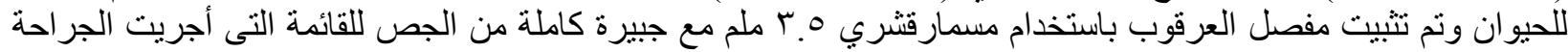

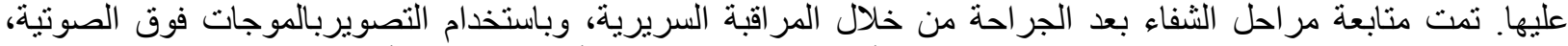

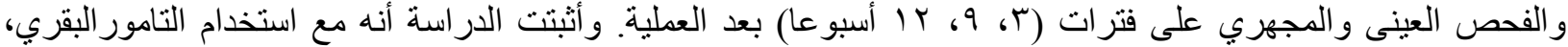

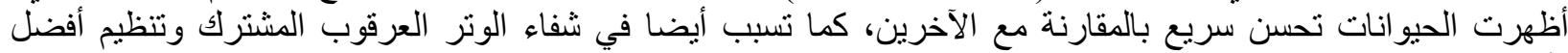

